\title{
Correction to: Deep Transfer Learning for Image Emotion Analysis: Reducing Marginal and Joint Distribution Discrepancies Together
}

\section{Yuwei $\mathrm{He}^{1}$ (D) Guiguang Ding ${ }^{1}$}

Published online: 6 January 2020

(c) Springer Science+Business Media, LLC, part of Springer Nature 2020

\section{Correction to: Neural Processing Letters https://doi.org/10.1007/s11063-019-10035-7}

The original article contains two mistakes:

1. The affiliations should be "School of Software; Beijing National Research Center for Information Science and Technology (BNRist), Tsinghua University, Beijing, China" rather than "Tsinghua University School of Life Sciences, Beijing, People's Republic of China".

2. The funding information should be "National Key Rl\&D Program of China (2018YFC0807500)" rather than empty.

Moreover, in the first paragraph of the 3.2 section in the paper, we think the presentation here is somewhat redundant. So we updated it as:

MMD can be represented as the distance in reproducing kernel Hilbert space [4]. As Euclidean space $\mathcal{V}$ is a finite vector space, Hilbert space is typically viewed as an infinite function space $\mathcal{H}$ and its orthogonal basis can be denoted as $\left\{\psi_{i}\right\}_{i=1}^{\infty}$, where $\psi_{i}$ is the base function in each dimension. If $\mathbf{X}$ is a random variable in domain $\boldsymbol{\Omega}$, a function $f: \boldsymbol{\Omega} \rightarrow \mathbb{R}$ in $\mathcal{H}$ can be presented as $\left(f_{1}, f_{2}, \ldots\right)_{\mathcal{H}}^{T}$ and $f(\mathbf{x})=\sum_{i=1}^{\infty} f_{i} \psi_{i}(\mathbf{x})$. We define another infinitedimensional feature map $\phi(\mathbf{x})$ in $\mathcal{H}$ as $\left(\psi_{1}(\mathbf{x}), \psi_{2}(\mathbf{x}), \ldots\right)_{\mathcal{H}}^{T}[3,21]$.

We find that:

$$
\langle f, \phi(\mathbf{x})\rangle=\sum_{i=1}^{\infty} f_{i} \psi_{i}(\mathbf{x})=f(\mathbf{x}) .
$$

The original article can be found online at https://doi.org/10.1007/s11063-019-10035-7.

$\bowtie$ Yuwei He

hyw16@mails.tsinghua.edu.cn

Guiguang Ding

dinggg@tsinghua.edu.cn

1 School of Software, Beijing National Research Center for Information Science and Technology (BNRist), Tsinghua University, Beijing, China 
Publisher's Note Springer Nature remains neutral with regard to jurisdictional claims in published maps and institutional affiliations. 